\title{
A tool to predict disparities in the timeliness of surgical treatment for breast cancer patients in the USA
}

\author{
Christopher G. Verdone ${ }^{1}$. Jennifer A. Bayron ${ }^{1} \cdot$ Cecilia Chang ${ }^{2} \cdot$ Chihsiung E. Wang $^{2}$ - Elin R. Sigurdson ${ }^{1}$. \\ Allison A. Aggon ${ }^{1} \cdot$ Andrea Porpiglia $^{1} \cdot$ Maureen V. Hill ${ }^{1} \cdot$ Mary T. Pronovost $^{3} \cdot$ Richard J. Bleicher $^{1}$ (1)
}

Received: 28 July 2021 / Accepted: 15 November 2021 / Published online: 11 January 2022

(c) The Author(s), under exclusive licence to Springer Science+Business Media, LLC, part of Springer Nature 2021

\begin{abstract}
Purpose Breast cancer outcomes are impaired by both delays and disparities in treatment. This study was performed to assess their relationship and to provide a tool to predict patient socioeconomic factors associated with risk for delay.

Methods The National Cancer Database was reviewed between 2004 and 2017 for patients with non-metastatic breast cancer managed with upfront surgery. Times to treatment were measured from the date of diagnosis. Patient, tumor, and treatment factors were assessed with attention paid to sociodemographic variables.

Results 514,187 patients remained after exclusions, with 84.3\% White, 10.8\% Black, 3.7\% Asian, and Hispanics comprising $5.6 \%$ of the cohort. Medicaid and uninsured patients had longer mean adjusted time to surgery ( $\geq 46$ days) versus private (36.7 days), Medicare (35.9 days), or other governmental insurance (39.8 days). After adjustment, Black race and Hispanic ethnicity were most impactful, adding 6.0 and 6.4 preoperative days, 10.9 and 11.5 days to chemotherapy, 11.1 and 9.1 days to radiation, and 12.5 and 8.9 days to endocrine therapy, respectively. Income, education, and insurance, among other factors, also affected delay. A nomogram, including race and sociodemographic factors, was created to predict the risk of preoperative delay.

Conclusion Significant disparities exist in timeliness of care for factors, including but not limited to, race and ethnicity. Although exact causes cannot be discerned, these data indicate population subsets whose intervals of care risk being longer than those specified by national quality standards. The nomogram created here may help direct resources to those at highest risk of incurring a treatment delay.
\end{abstract}

Keywords Diversity $\cdot$ Equity and inclusion $\cdot$ Disparities $\cdot$ Socioeconomic status $\cdot$ Delays $\cdot$ Risk factors $\cdot$ Breast cancer

This manuscript has been presented, in part, at the Virtual American Society of Breast Surgeons 22nd Annual Meeting April 29-May 2, 2021.

Richard J. Bleicher

richard.bleicher@fccc.edu

1 Department of Surgical Oncology, Fox Chase Cancer Center, 333 Cottman Avenue, Room C-308, Philadelphia, PA 19111, USA

2 Department of Surgery, NorthShore University Health System, Evanston, IL, USA

3 Department of Surgery, Lewis Katz School of Medicine, Temple University, Philadelphia, PA, USA

\section{Introduction}

Breast cancer is the most common malignancy in women. Disparities in breast cancer care are widely documented and associated socioeconomic and demographic factors have been the focus of many studies [1-6]. The precise reasons for differences in care and outcomes are felt to be myriad. Access to care and insurance status are just a few of the socioeconomic factors that have been shown to contribute to racial differences in outcomes for breast cancer [4]. It is also known that biology may contribute to the disparities in survival by race [5]. Treatment delays, too, have been associated with patient, demographic, and treatment factors $[3,4,6]$.

Although many factors are well documented to affect survival in patients with breast cancer, the timeliness of treatment has recently been a research focus to determine its 
effects on outcomes. We have previously found that both disease specific and overall survival are adversely affected by greater times between diagnosis and surgery, with overall survival declining by $9-10 \%$ per month of delay and breast cancer-specific mortality increasing by a relative $26 \%$ for each 60-day delay. While not a focus of that study, we found that minorities had greater times to surgery, suggesting that this could be a contributor to the disparities seen elsewhere [1].

This analysis was performed to clarify that observation and to assess, in detail, the differences in the timeliness of local and systemic therapies given for breast cancer in the USA for those managed with upfront surgery. In order to provide additional practical information to combat such disparities, we created a nomogram to best predict individuals at greatest risk for preoperative delay. There is no study, to our knowledge, that allows accurate prediction of delay risk for any particular patient. Identifying those at greatest risk for longer preoperative intervals may assist the direction of limited institutional and community resources.

\section{Methods}

After obtaining approval from the American College of Surgeons, de-identified data were retrieved from the National Cancer Database (NCDB) from between 2004 and 2017. The NCDB, a joint program of the Commission on Cancer $(\mathrm{CoC})$, the American College of Surgeons, and the American Cancer Society, is an accumulation of nationally standardized data collected from over $1500 \mathrm{CoC}$-accredited programs at the state, regional, and national level [7] and which captures approximately $70 \%$ of all new cancer diagnoses nationally. At the time of NCDB approval, the Fox Chase Cancer Center IRB deemed NCDB studies as exempt due to their de-identification. The selection map was based on predetermined patient, tumor, and treatment factors felt to be contributors and all found in the NCDB Participant User File 2017 Data Dictionary. We reviewed patients diagnosed with breast cancer managed with surgery first. Times to treatment were measured from the date of diagnosis. Patients whose age, sex, race, ethnicity, income, insurance, education, urban/ rural setting, and distance to facility were unknown were excluded from our analysis. Patients with distant metastatic disease and/or histology that was not defined were excluded (Fig. 1).

The cohort was limited to patients who underwent surgery as their initial treatment for homogeneity and to limit confounding related to differing sequences of treatment. Individuals undergoing neoadjuvant therapy, no surgery, or no treatment were therefore excluded. Patients were limited to those having their breast cancer as their first cancer diagnosis to avoid heterogeneity in the times to treatment that may occur when a patient experiences a recurrence, has residual treatment that may be ongoing, and for those who are already established with providers whose ease of access may differ. All patients who had surgery were included, except for those whose timing was unknown. Patients whose treatment timing and sequence from diagnosis through surgery, chemotherapy, radiation, immunotherapy, and endocrine therapies was unknown were excluded.

Adjusted mean and median time to respective treatments, surgery, chemotherapy, radiation, and hormone therapy were computed. All factors were adjusted for age, sex, race, ethnicity, income, insurance status, education, urban/rural location, distance to facility, Charlson Comorbidity Index, histology, grade, clinical stage, and pathologic stage. Due to a skewed distribution of times from diagnosis to respective treatments, multivariable regression models were carried out with log-transformed outcome in days to estimate delay based on each of the patient and tumor factors. Differences in estimated times to treatment, in days, were calculated relative to the predetermined category referent. Statistical analysis was performed using SAS 9.4 (SAS Institute Inc., Cary, NC). All tests were two-sided, and $p$ values $<0.05$ were considered statistically significant.

A nomogram was created to serve as a tool to predict delays to surgery based on the logistics regression model. Delay was defined for the nomogram as time $>60$ days to improve power and identify patients approaching the clinically significant 90 -day proposed national quality measure [1]. In order to assign point values to the patient variables, using the estimated regression coefficients, the predicted effects were ranked disregarding statistical significance and direction. The strongest predictor was determined and, sequentially, the other predictive factors were assigned point values, proportionally, relative to the most impactful variable. The nomogram was evaluated by calculating the area under the receiver operating characteristics curve (AUC). Data were split into $70 \%$ training and $30 \%$ testing sets to examine the predictive performance of the tool. The nomogram (Fig. 2) was validated, internally in the training set and externally in the validation set, by the area under the receiver operating characteristics (ROC) curve with AUCs of 0.65 and 0.64 , respectively (Supplemental Fig. S1).

\section{Results}

There were 514,187 patients after exclusions, with $99.3 \%$ being female. $84.3 \%$ were White, $10.8 \%$ Black, and 3.7\% Asian. Hispanics comprised $5.6 \%$ of the cohort. Fifteen percent had a median household income of $\angle \$ 40,277$, while $41.7 \%$ made $\geq \$ 63,333$. There were $7.4 \%$ of patients that were either Medicaid insured or uninsured, while $1.3 \%$ lived 
Fig. 1 Cohort inclusion and exclusion criteria-STROBE diagram
Inclusion Criteria:

- Patients with breast cancer $(\mathrm{n}=2,981,828)$

Patient Exclusions:

- Unknown sex or age $<18(\mathrm{n}=96)$

- Unknown race or ethnicity $(\mathrm{n}=165,383)$

- Unknown \% without high school diploma quartile $(\mathrm{n}=270,882)$

- Unknown primary payor (insurance status) or income $(n=52,324)$

- Unknown urban/rural status or great circle distance $(n=55,678)$

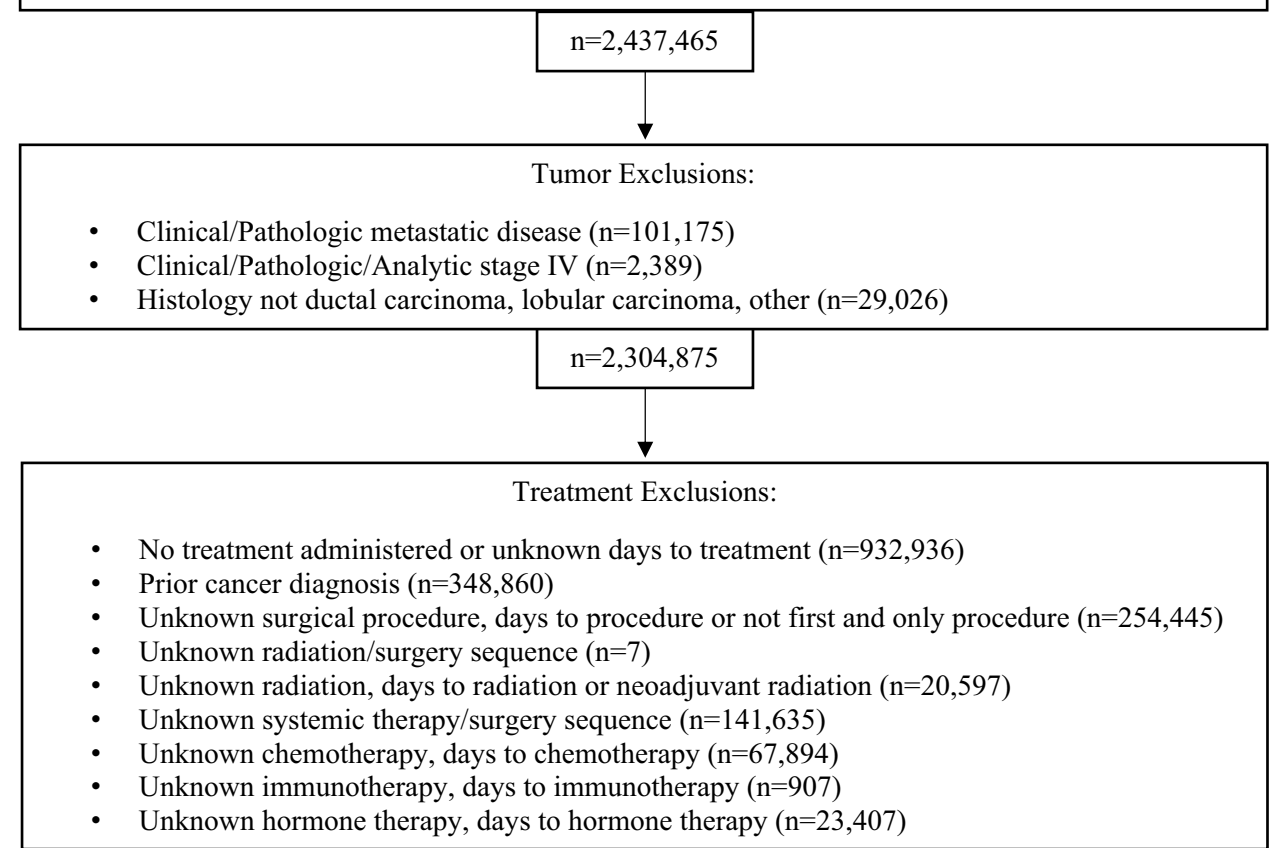
$\mathrm{n}=514,187$ in a rural area. Only 5.6\% lived $>50$ miles from their treatment facility (Table 1).

Table 2 and Supplemental Table 1 demonstrate the mean and median times (in days) to each treatment modality by demographic factor adjusted for age, sex, race, ethnicity, income, insurance status, education, urban/rural location, distance to facility, Charlson Comorbidity Index, histology, grade, clinical stage, and pathologic stage. The mean adjusted time from diagnosis to surgery for all patients in the cohort was 37.1 days. White patients experienced the shortest mean times to surgery relative to those of different races and non-Spanish/non-Hispanic patients had shorter times than those of Spanish/Hispanic ethnicity. Patients with no insurance or Medicaid were associated with a mean of 46 days or longer from diagnosis to surgery. Communities with lower high school graduation rates, those in metro areas, and those $>75$ miles from their point of care all had the greatest time from diagnosis to surgery realized in their respective categories (Table 2). Time from diagnosis to chemotherapy, radiation, and hormone therapy demonstrated the same relationships (Supplemental Table 1).

Time from diagnosis to surgery was $>30$ days in $52.7 \%$ of the cohort $(n=270,706)$. There were 62,709 patients (12.2\%) that realized a time from diagnosis to surgical treatment $>60$ days and $3.3 \%$ of patients experienced a delay $>90$ days. In other words, $26.6 \%$ of those experiencing time to surgery $>60$ days ultimately underwent surgical treatment $>90$ days from diagnosis.

Multivariable analysis with log-transformed outcomes (in days) are displayed in Table 3 and Supplemental Table 2, relative to the referent categories. These log-transformed outcomes represent the number of additional or fewer days that this variable's category is associated with relative to that variable's referent category. Time to surgery and chemotherapy was predicted significantly shorter for White as compared to Non-White patients. The greatest disparity was seen for Black versus White patients with additional days to surgery, chemotherapy, radiation, and endocrine treatments being 6.0,10.9, 11.1, and 12.5 (all $p$ 's $<0.0001$ ), 


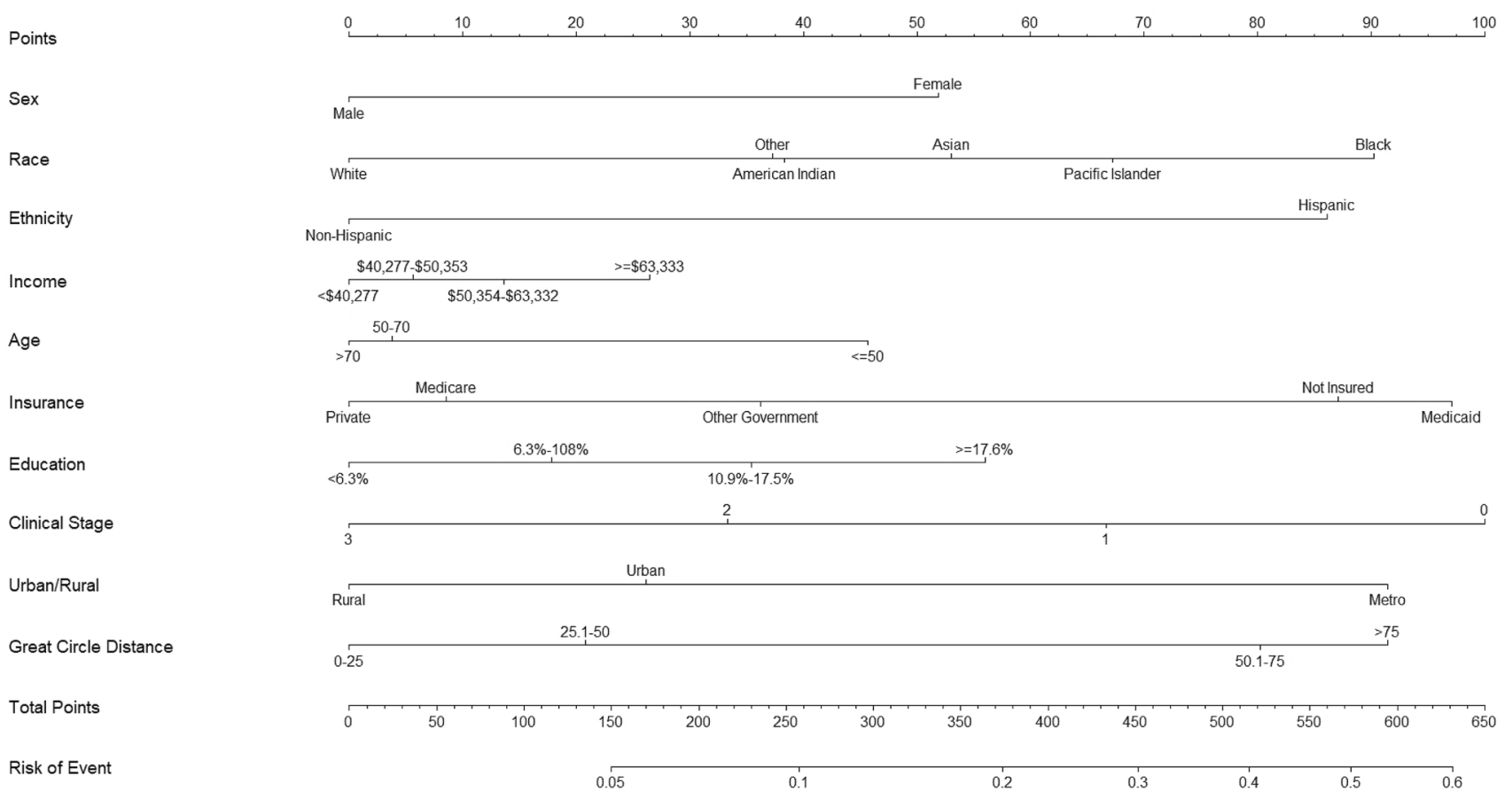

Fig. 2 Nomogram for risk of time from diagnosis to surgery $>60$ days. Each patient demographic category has variables with corresponding point values. Once the points for a patient's factors are summed, the total point value has an associated risk of event value.

respectively. Hispanic ethnicity as well as living in areas with a lower percentage of high school graduates was associated with significant delays to all measured therapies. Medicaid patients or those who were not insured had longer mean adjusted times to surgery ( $\geq 46$ days) versus private (36.7 days), Medicare (35.9 days), or other governmental insurance (39.8 days). Likewise, the Medicaid patients and those without insurance also realized significantly greater estimated time intervals to surgery relative to patients in the other aforementioned insurance categories. There was a delay to surgery associated with higher median household income (all $p$ ' $s<0.0001$ ). Living a distance $>50$ miles from their treatment facility, relative to $<25$ miles, was associated with a $\geq 5$.6-day delay to surgery and chemotherapy administration. Metropolitan setting was also associated with significant delays to local and systemic therapies. Lobular carcinoma diagnosis was associated with $>2$ additional days to surgery relative to ductal carcinoma diagnosis. Poorly differentiated tumor grade as well as more advanced clinical stage were significantly associated with shorter times to surgery (Table 3).

A nomogram (Fig. 2) was created to identify patients most at risk to realize a time $>60$ days from diagnosis to surgery. The AUCs for internal and external validation of the nomogram were 0.65 and 0.64 , respectively (Supplemental Fig. 1). Patient factors within each of the following
Risk of event is the probability of experiencing time from diagnosis to surgery $>60$ days. For example, a 0.6 risk of event portends a $60 \%$ likelihood of incurring a time from diagnosis to surgery $>60$ days

categories: sex, race, ethnicity, income, age, insurance, education (percentage of community without a high school diploma), clinical stage, urban-rural setting, and great circle distance (distance from home to treating facility) each confer points. The point values per patient factor are noted along the $\mathrm{X}$-axis for each variable along the $\mathrm{Y}$-axis. A summation of those points is then plotted on the total points line at the bottom of the nomogram where the corresponding risk directly below that value is shown for a preoperative delay of $>60$ days. This risk of event calculated is a probability, so a patient with a risk score of 0.6 corresponds to a $60 \%$ likelihood of incurring a delay from diagnosis to surgery $>60$ days.

\section{Discussion}

Disparities in breast cancer care are widely documented and multifactorial. Contributions of sociodemographic, economic, and biologic factors to treatment delays and breast cancer outcomes have been the subject of prior investigations [1-6, 8-11]. A 2019 review by Coughlin evaluated neighborhood disadvantage, racial discrimination, immigration status, and social support for patients with breast cancer and found that social determinants of health play 
Table 1 Cohort demographics

\begin{tabular}{|c|c|c|}
\hline & \multicolumn{2}{|l|}{ Total } \\
\hline & $n$ & $\%$ \\
\hline Total patients & 514,187 & 100.0 \\
\hline \multicolumn{3}{|l|}{ Age (years) } \\
\hline$\leq 50$ & 112,029 & 21.8 \\
\hline $50-70$ & 290,809 & 56.6 \\
\hline$>70$ & 111,349 & 21.7 \\
\hline \multicolumn{3}{|l|}{ Sex } \\
\hline Male & 3552 & 0.7 \\
\hline Female & 510,635 & 99.3 \\
\hline \multicolumn{3}{|l|}{ Race } \\
\hline White & 433,625 & 84.3 \\
\hline Black & 55,383 & 10.8 \\
\hline American Indian/Aleutian/Eskimo & 1436 & 0.3 \\
\hline Asian & 18,887 & 3.7 \\
\hline Hawaiian/Pacific Islander & 426 & 0.1 \\
\hline Other & 4430 & 0.9 \\
\hline \multicolumn{3}{|l|}{ Ethnicity } \\
\hline Non-Spanish/Non-Hispanic & 485,630 & 94.5 \\
\hline Spanish/Hispanic & 28,557 & 5.6 \\
\hline \multicolumn{3}{|l|}{ Income } \\
\hline$<\$ 40,277$ & 77,120 & 15.0 \\
\hline$\$ 40,277-\$ 50,353$ & 102,471 & 19.9 \\
\hline$\$ 50,354-\$ 63,332$ & 120,072 & 23.4 \\
\hline$\geq \$ 63,333$ & 214,524 & 41.7 \\
\hline \multicolumn{3}{|l|}{ Insurance } \\
\hline Uninsured & 8279 & 1.6 \\
\hline Private insurance & 283,716 & 55.2 \\
\hline Medicaid & 29,676 & 5.8 \\
\hline Medicare & 186,813 & 36.3 \\
\hline Other government insurance & 5703 & 1.1 \\
\hline \multicolumn{3}{|l|}{ Percent without high school diploma } \\
\hline$\geq 17.6 \%$ & 87,544 & 17.0 \\
\hline $10.9 \%-17.5 \%$ & 122,322 & 23.8 \\
\hline $6.3 \%-10.8 \%$ & 149,974 & 29.2 \\
\hline$<6.3 \%$ & 154,347 & 30.0 \\
\hline \multicolumn{3}{|l|}{ Urban/rural status } \\
\hline Metro & 448,630 & 87.3 \\
\hline Urban & 58,718 & 11.4 \\
\hline Rural & 6839 & 1.3 \\
\hline \multicolumn{3}{|l|}{ Great circle distance (miles) } \\
\hline $0-25.0$ & 432,889 & 84.2 \\
\hline $25.1-50.0$ & 52,881 & 10.3 \\
\hline $50.1-75.0$ & 14,671 & 2.9 \\
\hline$>75.0$ & 13,746 & 2.7 \\
\hline
\end{tabular}

an important role in both stage at diagnosis and eventual outcome [2]. Because the interplay of sociodemographic and economic factors with treatment delay is complex, the nomogram presented here provides a novel clinical tool to help identify those most likely to incur delays to surgical treatment.

Sociodemographic and economic patient factors are often found to be linked to treatment delays. Smith et al. in 2013 evaluated treatment delays in women aged 15-39 and found a significant delay of $>6$ weeks among Hispanic and African American patients as compared to non-Hispanic Whites. Lower socioeconomic status and patients with either Medicaid, other government-assisted insurance, or a lack of insurance were found to have a significantly greater likelihood of a $>6$-week delay between diagnosis and treatment [3]. Reeder-Hayes et.al demonstrated on review of 2559 breast cancer patients in the Carolina Breast Center Study that Black women had 1.73 times higher odds of initiating treatment $>60$ days from diagnosis relative to White women [6]. A 2020 retrospective review of racial differences in time to breast cancer surgery found that even within the US Military Health System, where insurance and access should be uniform across beneficiaries, there was a significantly greater time to surgery for Non-Hispanic Black women relative to Non-Hispanic White women [4]. It is therefore not surprising that we found that Black race and Hispanic ethnicity were each associated with a 6-day delay between diagnosis and surgery when compared to White and Non-Hispanic patients, respectively.

Differences in survival and the likelihood of an early stage of diagnosis by race and ethnicity have also been shown to be partially attributable to tumor biologic factors [5]. Triple-negative breast cancer (TNBC) has an incidence in African American women that is twice that for White women and has been found to have a younger age distribution than other subtypes [10]. Success in directing resources to eliminate disparities will only be achieved when we have a complete understanding of the influence that social, economic, demographic, environmental, and biologic factors have on breast cancer outcomes.

Although times to each subspecialty treatment have been presented for the reader, in this study, we chose to focus our evaluation predominantly on delays from diagnosis to surgery because timeliness in initiating treatment represents one of the largest hurdles in receiving care for all patients and may be even more challenging for disadvantaged groups and those with poor healthcare access. This interval is also important because a longer time between diagnosis and surgery is associated with poorer outcomes. Like treatment delay disparity, breast cancer survival has also been linked to socioeconomics, race, education, poverty, and health insurance status $[2-4,6]$. We found that the mean adjusted time to surgery in our cohort was 37.1 days, which, fortunately, is far shorter than the 90-day proposed standard [1]. Polverini and colleagues found in an NCDB cohort that patients with stage I and II breast cancers realized a lower overall survival when time to surgery exceeded 12 weeks [12]. We have also 
Table 2 Unadjusted and adjusted time to surgery by demographic factor

\begin{tabular}{|c|c|c|c|c|}
\hline & \multicolumn{4}{|c|}{ Diagnosis to Surgery } \\
\hline & $\begin{array}{l}\text { Unadjusted } \\
\text { mean (days) }\end{array}$ & $\begin{array}{l}\text { Unadjusted } \\
\text { median (days) }\end{array}$ & $\begin{array}{l}\text { Adjusted } \\
\text { mean (days) }\end{array}$ & $\begin{array}{l}\text { Adjusted } \\
\text { median } \\
\text { (days) }\end{array}$ \\
\hline Total patients & 37.5 & 27.3 & 37.1 & 35.8 \\
\hline \multicolumn{5}{|l|}{ Age (years) } \\
\hline$\leq 50$ & 40.8 & 35.0 & 40.2 & 39.1 \\
\hline $50-70$ & 36.9 & 31.0 & 36.5 & 35.1 \\
\hline$>70$ & 35.9 & 30.0 & 35.6 & 34.5 \\
\hline \multicolumn{5}{|l|}{ Sex } \\
\hline Male & 32.6 & 27.0 & 32.0 & 30.6 \\
\hline Female & 37.5 & 32.0 & 37.2 & 35.8 \\
\hline \multicolumn{5}{|l|}{ Race } \\
\hline White & 36.4 & 31.0 & 36.0 & 34.9 \\
\hline Black & 44.7 & 36.0 & 44.4 & 43.4 \\
\hline American Indian/Aleutian/Eskimo & 40.1 & 33.0 & 39.4 & 38.6 \\
\hline Asian & 41.4 & 35.0 & 40.9 & 39.9 \\
\hline Hawaiian/Pacific islander & 43.8 & 36.0 & 43.5 & 42.8 \\
\hline Other & 43.5 & 36.0 & 43.0 & 42.8 \\
\hline \multicolumn{5}{|l|}{ Ethnicity } \\
\hline Non-Spanish/Non-Hispanic & 37.0 & 32.0 & 36.6 & 35.4 \\
\hline Spanish/Hispanic & 46.5 & 39.0 & 46.3 & 45.4 \\
\hline \multicolumn{5}{|l|}{ Income } \\
\hline$<\$ 40,277$ & 39.1 & 33.0 & 38.8 & 38.3 \\
\hline$\$ 40,277-\$ 50,353$ & 37.2 & 31.0 & 37.0 & 35.6 \\
\hline$\$ 50,354-\$ 63,332$ & 37.2 & 32.0 & 36.8 & 35.5 \\
\hline$\geq \$ 63,333$ & 37.3 & 32.0 & 36.8 & 35.4 \\
\hline \multicolumn{5}{|l|}{ Insurance } \\
\hline Uninsured & 46.5 & 38.0 & 46.0 & 46.1 \\
\hline Private insurance & 37.1 & 32.0 & 36.7 & 35.7 \\
\hline Medicaid & 47.0 & 39.0 & 46.6 & 46.4 \\
\hline Medicare & 36.2 & 30.0 & 35.9 & 34.7 \\
\hline Other government insurance & 39.9 & 34.0 & 39.8 & 38.8 \\
\hline \multicolumn{5}{|l|}{ Percent without high school diploma } \\
\hline$\geq 17.6 \%$ & 40.8 & 34.0 & 40.5 & 40.2 \\
\hline $10.9-17.5 \%$ & 37.9 & 32.0 & 37.7 & 36.4 \\
\hline $6.3-10.8 \%$ & 36.9 & 32.0 & 36.4 & 35.2 \\
\hline$<6.3 \%$ & 36.0 & 31.0 & 35.5 & 34.4 \\
\hline \multicolumn{5}{|l|}{ Urban/Rural status } \\
\hline Metro & 38.1 & 33.0 & 37.7 & 36.2 \\
\hline Urban & 33.7 & 29.0 & 33.5 & 32.8 \\
\hline Rural & 32.7 & 28.0 & 32.0 & 31.5 \\
\hline \multicolumn{5}{|l|}{ Great circle distance (miles) } \\
\hline $0-25.0$ & 37.5 & 32.0 & 37.1 & 35.5 \\
\hline $25.1-50.0$ & 36.7 & 31.0 & 36.5 & 35.9 \\
\hline $50.1-75.0$ & 38.7 & 33.0 & 38.7 & 38.1 \\
\hline$>75.0$ & 39.9 & 34.0 & 39.6 & 39.0 \\
\hline
\end{tabular}

Values enumerated have been adjusted for age, sex, race, ethnicity, income, insurance status, education, urban/rural location, distance to facility, Charlson comorbidity index, histology, grade, clinical stage, and pathologic stage 
Table 3 Multivariable analysis of time from diagnosis to surgery with log-transformed outcomes (in days)

\begin{tabular}{|c|c|c|c|c|}
\hline \multirow{3}{*}{ Age (years) } & \multicolumn{4}{|c|}{ Diagnosis to surgery } \\
\hline & \multirow{2}{*}{$\begin{array}{l}\text { Estimated days * } \\
-\end{array}$} & \multicolumn{2}{|c|}{$95 \%$ Confidence Interval } & \multirow{2}{*}{$\frac{p \text { value }}{-}$} \\
\hline & & - & - & \\
\hline$\leq 50$ (ref) & - & - & & \\
\hline $50-70$ & -2.3 & -2.4 & -2.2 & $<0.0001$ \\
\hline$>70$ & -2.7 & -2.9 & -2.6 & $<0.0001$ \\
\hline \multicolumn{5}{|l|}{ Sex } \\
\hline Male (ref) & - & - & - & - \\
\hline Female & 6.1 & 5.1 & 7.1 & $<0.0001$ \\
\hline \multicolumn{5}{|l|}{ Race } \\
\hline White (ref) & - & - & - & - \\
\hline Black & 6.0 & 5.6 & 6.5 & $<0.0001$ \\
\hline American Indian/Aleutian/Eskimo & 1.3 & 0.1 & 2.5 & 0.0305 \\
\hline Asian & 2.8 & 2.4 & 3.2 & $<0.0001$ \\
\hline Hawaiian/Pacific Islander & 3.5 & 1.3 & 5.9 & 0.0014 \\
\hline Other & 1.6 & 0.9 & 2.4 & $<0.0001$ \\
\hline \multicolumn{5}{|l|}{ Ethnicity } \\
\hline Non-Spanish/Non-Hispanic (ref) & - & - & - & - \\
\hline Spanish/Hispanic & 6.4 & 5.9 & 6.9 & $<0.0001$ \\
\hline \multicolumn{5}{|l|}{ Income } \\
\hline$<\$ 40,277$ (ref) & - & - & - & - \\
\hline$\$ 40,277-\$ 50,353$ & 0.9 & 0.6 & 1.1 & $<0.0001$ \\
\hline$\$ 50,354-\$ 63,332$ & 1.5 & 1.3 & 1.8 & $<0.0001$ \\
\hline$\geq \$ 63,333$ & 2.5 & 2.2 & 2.9 & $<0.0001$ \\
\hline \multicolumn{5}{|l|}{ Insurance } \\
\hline Uninsured (ref) & - & - & - & - \\
\hline Private insurance & -3.9 & -4.2 & -3.5 & $<0.0001$ \\
\hline Medicaid & 1.5 & 1.0 & 2.2 & $<0.0001$ \\
\hline Medicare & -3.5 & -3.8 & -3.1 & $<0.0001$ \\
\hline Other government insurance & -2.0 & -2.6 & -1.3 & $<0.0001$ \\
\hline \multicolumn{5}{|l|}{ Percent without high school diploma } \\
\hline$\geq 17.6 \%$ (ref) & - & - & - & - \\
\hline $10.9-17.5 \%$ & -0.9 & -1.1 & -0.7 & $<0.0001$ \\
\hline $6.3-10.8 \%$ & -1.8 & -1.9 & -1.6 & $<0.0001$ \\
\hline$<6.3 \%$ & -2.7 & -2.8 & -2.5 & $<0.0001$ \\
\hline \multicolumn{5}{|l|}{ Urban/Rural status } \\
\hline Metro (ref) & - & - & - & - \\
\hline Urban & -4.4 & -4.5 & -4.4 & $<0.0001$ \\
\hline Rural & -6.5 & -6.7 & -6.2 & $<0.0001$ \\
\hline \multicolumn{5}{|l|}{ Great circle distance (miles) } \\
\hline $0-25.0$ (ref) & - & - & - & - \\
\hline $25.1-50.0$ & 1.7 & 1.4 & 2.0 & $<0.0001$ \\
\hline $50.1-75.0$ & 5.6 & 5.1 & 6.3 & $<0.0001$ \\
\hline$>75.0$ & 5.9 & 5.3 & 6.5 & $<0.0001$ \\
\hline \multicolumn{5}{|l|}{ Charlson comorbidity index } \\
\hline 0 (ref) & - & - & - & - \\
\hline 1 & 0.5 & 0.3 & 0.7 & $<0.0001$ \\
\hline 2 & 2.0 & 1.6 & 2.5 & $<0.0001$ \\
\hline 3 & 3.7 & 3.0 & 4.5 & $<0.0001$ \\
\hline \multicolumn{5}{|l|}{ Histology } \\
\hline Ductal (ref) & - & - & - & - \\
\hline
\end{tabular}


Table 3 (continued)

\begin{tabular}{|c|c|c|c|c|}
\hline \multirow[b]{3}{*}{ Lobular } & \multicolumn{4}{|c|}{ Diagnosis to surgery } \\
\hline & \multirow{2}{*}{$\begin{array}{l}\text { Estimated days * } \\
2.2\end{array}$} & \multicolumn{2}{|c|}{ 95\% Confidence Interval } & \multirow{2}{*}{$\begin{array}{c}p \text { value } \\
<0.0001\end{array}$} \\
\hline & & 1.9 & 2.6 & \\
\hline Other/unknown & -1.1 & -1.8 & -0.3 & 0.0095 \\
\hline \multicolumn{5}{|l|}{ Grade } \\
\hline Well differentiated (ref) & - & - & - & - \\
\hline Moderately differentiated & 0.2 & 0.1 & 0.4 & 0.0029 \\
\hline Poorly differentiated & -1.3 & -1.4 & -1.1 & $<0.0001$ \\
\hline Undifferentiated/anaplastic & -0.8 & -1.6 & 0.1 & 0.0882 \\
\hline \multicolumn{5}{|l|}{ Clinical stage } \\
\hline 0 (ref) & - & - & - & - \\
\hline 1 & -5.6 & -5.7 & -5.4 & $<0.0001$ \\
\hline 2 & -5.1 & -5.3 & -4.8 & $<0.0001$ \\
\hline 3 & -7.2 & -7.5 & -6.8 & $<0.0001$ \\
\hline \multicolumn{5}{|l|}{ Pathologic stage } \\
\hline 0 (ref) & - & - & - & - \\
\hline 1 & 0.7 & 0.2 & 1.1 & 0.0022 \\
\hline 2 & 1.4 & 0.9 & 1.9 & $<0.0001$ \\
\hline 3 & 3.0 & 2.4 & 3.7 & $<0.0001$ \\
\hline
\end{tabular}

Estimated days to surgery are enumerated for each variable, relative to a referent category. For example, Black race is associated with 6 additional days from diagnosis to surgery relative to White patients

Ref Referent category

*Relative to referent category

previously found, when evaluating both Surveillance, Epidemiology, and End Results (SEER)-Medicare-linked database and NCDB breast cancer cohorts, that time to surgery was significantly associated with overall survival for stage I and II disease in both datasets. In that study, a 3.1-4.6\% absolute decline in overall survival was seen with preoperative delays of 90-120 days [1]. That prior study also found that $>98 \%$ of patients in the USA have surgery within 90 days of diagnosis [1]. Although these studies suggested that an optimal maximum time from diagnosis to surgery should be $<90$ days, we chose 60 days for this nomogram in order to provide a more aggressive time frame and to direct concern to a wider group of patients who may be at risk, especially as 1 in 4 patients who realized a time to surgery of $>60$ days had their surgical treatment $>90$ days from diagnosis.

The nomogram presented here includes sociodemographic factors and calculates a sum of contributions that estimates the specific risk of delay $>60$ days between diagnosis and surgery. The best use of this tool probably resides in using it to compare risks between patients. So, for example, if one were to calculate the individual risks of a set of new patients presenting to a clinic, the nomogram can objectively and uniformly identify specific patients at greatest risk of delay who might benefit from greater attention and follow-up using phone calls, scheduling efforts, or other measures, to ensure that their care is not delayed. As can be seen from the number of points contributed by each factor in the nomogram, some carry a greater weight for incurring delay and may be predictable, such as Black race, Hispanic ethnicity, coverage by Medicaid, and a larger distance from the treating facility, while others are not as intuitive. For instance, we found that higher income predicted longer preoperative intervals. Although the reasons are uncertain, it may be explained by greater access to second opinions, which are associated with delays [13]. Similarly, the association of lower clinical stage with delays may reflect less urgency on the part of providers to expedite such patients. Individuals in metropolitan locations may experience delays because the institutions in those locations care for greater numbers of patients relative to resources, limiting their ability to schedule and treat them expeditiously.

Our modeling suggests that resources to curb delays may positively impact greater patient numbers when they are focused on minorities and those with lower education levels, Medicaid or no insurance, and those at greatest distance from their treatment facilities. Strategies to mitigate delays to surgical treatment will vary on the locoregional and institutional level based on resources and patient population, but this national dataset provides a framework from which regional differences can be refined.

The need for improvements in the preoperative period stem not just from their potential to improve outcomes, but because times to surgery have been noted to be increasing [14]. Preoperative delays are also associated with delays in 
other treatment modalities, each of which have their own timeliness standards in universally adopted quality measures [15]. The universally accepted standard for time from diagnosis to treatment is 120 days for chemotherapy and 365 days for both radiation and endocrine therapies $[16,17]$. While not the focus of our study, we have presented the times from diagnosis to chemotherapy, radiation, and endocrine therapies (Supplemental Tables 1 and 2) for completeness. The mean adjusted times from diagnosis to chemotherapy, radiotherapy, and endocrine therapy were 86.4 days, 120.7 days, and 154.9 days, respectively, demonstrating a high degree of compliance with quality measures overall [16]. We also found that the time to radiation and endocrine therapy significantly increased for patients having tumors of more advanced pathologic stage, which is expected and consistent with the fact that such patients are more likely to be recommended for adjuvant chemotherapy and have a more complicated evaluation and treatment plan. Although our analysis excluded patients receiving neoadjuvant chemotherapy because this was beyond the scope of our study, further evaluation of that cohort is needed.

There may be confounding patient factors that were not included in our analysis that contribute to how promptly patients receive treatment. Even the complexity of the surgery itself or skill of the operating surgeon can affect how promptly patients have their operative procedures, although this is not something typically available in any dataset. Longer cases necessitating more operating room block time may be more challenging to schedule [14]. Meanwhile, second opinions, the addition of supplementary imaging, and a need for multiple biopsies have also been found to lengthen the preoperative interval $[18,19]$. Even anxiety itself is associated with delays, although this cannot be captured in most datasets [20]. It must be noted that the Severe Acute Respiratory Syndrome Coronavirus-2 (SARS-CoV-2) pandemic of 2020, which brought with it widespread delays due to its massive disruption to the healthcare system and concessions to standard breast cancer management [21], occurred after our cohort data were collected and is therefore not accounted for by our nomogram. Although we know some of its effects on patient populations, the impact on minority and disadvantaged segments of society is not yet clear.

In addition to the confounding factors and considerations described above, there are further limitations that warrant discussion. The NCDB is a national dataset and so local or regional trends and practice patterns are not clearly discernable nor can they be determined by this or any national nomogram. Clinicians should keep this in mind when implementing the nomogram in practice, although this may serve as a starting point for individual institutions. We also should note that in constructing the tool, there were many patients that needed to be excluded for even a single unknown data point (Supplemental Table 3) in order to construct a valid, applicable nomogram. It is possible that there were certain patient variable combinations that were over- or under-represented in the nomogram due to this exclusion requirement, although as shown in Supplemental Table 3, proportionally, the various patient factor subsets are relatively unchanged pre- and post-exclusion.

The strengths of our study include a large population cohort, and although this study is a retrospective review, the data are prospectively collected and represent approximately $70 \%$ of newly diagnosed malignancies in the US population, as well as the combined experience of $\mathrm{CoC}$ centers. This dataset also documents how patients are treated and includes the associated biases that such individuals are subjected to in the real world. Unfortunately, although we are able to predict who is at risk for delay, the exact causes of delay cannot be discerned from the dataset. Confounders from any dataset, even one as large as the NCDB, do exist, and while the NCDB is felt to be generalizable to the population at large, there could be differences for individual subsets that limit the application of the nomogram. We plan to implement the nomogram within our health system. We hope that with application, further understanding of performance and opportunities to improve the tool will result.

In conclusion, disparities in the timeliness of treatment exist for minorities and those with socioeconomic disadvantages. These disparities may affect breast cancer outcomes in these groups and further attention is needed to limit the magnitude and effect of delay on breast cancer mortality. The nomogram created here has the potential to allow prospective and objective prediction of what patients may be most at risk for preoperative delay and improve compliance with national quality standards. We hope this tool permits providers to identify those at risk while paying attention to local practice patterns and resources. We believe that the nomogram should be used to help direct resources to those with the greatest risk to minimize such disparities.

Supplementary Information The online version contains supplementary material available at https://doi.org/10.1007/s10549-021-06460-9.

Acknowledgements The NCDB is a joint project of the Commission on Cancer of the American College of Surgeons and the American Cancer Society. The data used in the study are derived from a de-identified NCDB file. The American College of Surgeons and the Commission on Cancer have not verified and are not responsible for the analytic or statistical methodology employed or the conclusions drawn from these data by the investigators. This work was supported by United States Public Health Services grant P30CA006927 for analysis of the data via support of our biostatistics facility and by generous private donor support from the Marlyn Fein Chapter of the Fox Chase Cancer Center Board of Associates for analysis and interpretation of the data. 
Funding United States Public Health Services Grant P30CA006927 and private donor support from the Marlyn Fein Chapter of the Fox Chase Cancer Center Board of Associates.

Data availability The data analyzed and supporting this nomogram are available from the American College of Surgeons National Cancer Database (NCDB). Data are restricted and analyzed for this manuscript only after application and approval by the American College of Surgeons. Data are available upon reasonable request and approval of the American College of Surgeons.

Code availability SAS 9.4 (SAS Institute Inc., Cary, NC).

\section{Declarations}

Conflict of interest None to disclose.

Ethical approval At the time of NCDB approval, the Fox Chase Cancer Center IRB deemed NCDB studies as exempt due to their de-identification.

Consent to participate No patient consent to participate was required as study was performed on the NCDB databank after approval from the American College of Surgeons.

\section{References}

1. Bleicher RJ et al (2016) Time to surgery and breast cancer survival in the United States. JAMA Oncol 2(3):330-339

2. Coughlin SS (2019) Social determinants of breast cancer risk, stage, and survival. Breast Cancer Res Treat 177(3):537-548

3. Smith EC, Ziogas A, Anton-Culver H (2013) Delay in surgical treatment and survival after breast cancer diagnosis in young women by race/ethnicity. JAMA Surg 148(6):516-523

4. Eaglehouse YL et al (2019) Racial differences in time to breast cancer surgery and overall survival in the US military health system. JAMA Surg 154(3):e185113

5. Iqbal J et al (2015) Differences in breast cancer stage at diagnosis and cancer-specific survival by race and ethnicity in the United States. JAMA 313(2):165-173

6. Reeder-Hayes KE et al (2019) Race and delays in breast cancer treatment across the care continuum in the Carolina breast cancer study. Cancer 125(22):3985-3992

7. National Cancer Database Tools 2021 (2021). https://www.facs. org/quality-programs/cancer/ncdb. Accessed 19 Sep 2021
8. Hossain F et al (2019) Neighborhood social determinants of triple negative breast cancer. Front Public Health 7:18

9. Roseland ME et al (2017) Influence of clinical, societal, and treatment variables on racial differences in ER-/PR- breast cancer survival. Breast Cancer Res Treat 165(1):163-168

10. Newman LA, Kaljee LM (2017) Health disparities and triplenegative breast cancer in African American women: a review. JAMA Surg 152(5):485-493

11. DeGuzman PB et al (2017) Impact of urban neighborhood disadvantage on late stage breast cancer diagnosis in Virginia. J Urban Health 94(2):199-210

12. Polverini AC et al (2016) Time to treatment: measuring quality breast cancer care. Ann Surg Oncol 23(10):3392-3402

13. Bleicher RJ et al (2019) Treatment delays from transfers of care and their impact on breast cancer quality measures. Breast Cancer Res Treat 173(3):603-617

14. Bleicher RJ et al (2012) Preoperative delays in the US medicare population with breast cancer. J Clin Oncol 30(36):4485-4492

15. Bleicher RJ (2018) Timing and delays in breast cancer evaluation and treatment. Ann Surg Oncol 25(10):2829-2838

16. National Accreditation Program For Breast Centers Standards Manual. NAPBC Standards and Resources 2018. https://accre ditation.facs.org/accreditationdocuments/NAPBC/Portal\%20Res ources/2018NAPBCStandardsManual.pdf. Accessed 7 Feb 2021

17. Committee, C.o.C.Q.I. (2019) Commission on cancer quality measures. https://www.facs.org/-/media/files/qualityprograms/ cancer/ncdb/quality-measures.ashx. Accessed 9 July 2021

18. Bleicher RJ et al (2009) Association of routine pretreatment magnetic resonance imaging with time to surgery, mastectomy rate, and margin status. J Am Coll Surg 209(2):180-7 (quiz 294-5)

19. Hulvat $\mathrm{M}$ et al (2010) Time from diagnosis to definitive operative treatment of operable breast cancer in the era of multimodal imaging. Surgery 148(4):746-50 (discussion 750-1)

20. Ferrante JM, Chen PH, Kim S (2008) The effect of patient navigation on time to diagnosis, anxiety, and satisfaction in urban minority women with abnormal mammograms: a randomized controlled trial. J Urban Health 85(1):114-124

21. Dietz JR et al (2020) Recommendations for prioritization, treatment, and triage of breast cancer patients during the COVID-19 pandemic. The COVID-19 pandemic breast cancer consortium. Breast Cancer Res Treat 181(3):487-497

Publisher's Note Springer Nature remains neutral with regard to jurisdictional claims in published maps and institutional affiliations. 